Conference Proceedings Paper-Sensors and Applications

\title{
Sensorized Garments for Biomedical Monitoring: Design Issues
}

\author{
Giuseppe Andreoni *, Carlo Emilio Standoli and Paolo Perego \\ Politecnico di Milano, Design Dept., via G. Durando 38/A, 20158 Milan, Italy; \\ E-Mails: carloemilio.standoli@polimi.it (C.E.S.), paolo.perego@polimi.it (P.P.) \\ * Author to whom correspondence should be addressed; E-Mail: giuseppe.andreoni@polimi.it; \\ Tel.: +39-02-2399-5999; Fax: +39-02-2399-5860.
}

Published: 10 November 2015

\begin{abstract}
This paper discusses the user and technical requirements in designing smart garments for biomedical monitoring in several and very different applications: in hospital settings, during activities of daily living (ADL), sport and fitness, home care, working environments. Anthropometric and gender considerations are to be included into design as well as textile requirements like elasticity, washability and chemical agents effects for preserving sensors' efficacy and reliability, and assuring the proper duration of the product for the complete life cycle. Physiological issues are mainly due to skin conductance (and related operations: cleaning, scrubbing the external layer of dead skin cells, the presence of hair - especially in male subjects), skin tolerance and irritation, and the effect of sweat and perspiration. All these factors strongly affect the design and technical choices (materials in particular) but aesthetical requirements are proved to be crucial as well as comfort and wearability. For this aspect, user's age, target application, and fashion trend could not be ignored, because they determine the final success of the wearable monitoring approach.
\end{abstract}

Keywords: wearable systems; sensing garments; design; smart textiles; sensors;

\section{Introduction}

The 2015-2025 decade has been elected the "Wearable Era" for the diffusion and incidence of new miniaturized and wearable products and related services in our life. Most of these systems are dedicated at collecting and process data form our body and for this reason they are often considered Health related devices. Wearable Health Systems (WHS) or Wearable Biomedical Systems (WBS) are integrated systems on body-worn platforms like wrist-worn devices or even biomedical clothes, offering pervasive 
solutions for continuous health status monitoring though non-invasive biomedical, biochemical and physical measurements [1].

The main fields of application are Medicine, Life Style monitoring and Sport analysis (including fitness). The first device generation are a plurality of small devices to be worn but the arriving 2.0 generation tries to embed sensors and systems in our natural interface towards the external world: Our garments (Figure 1).

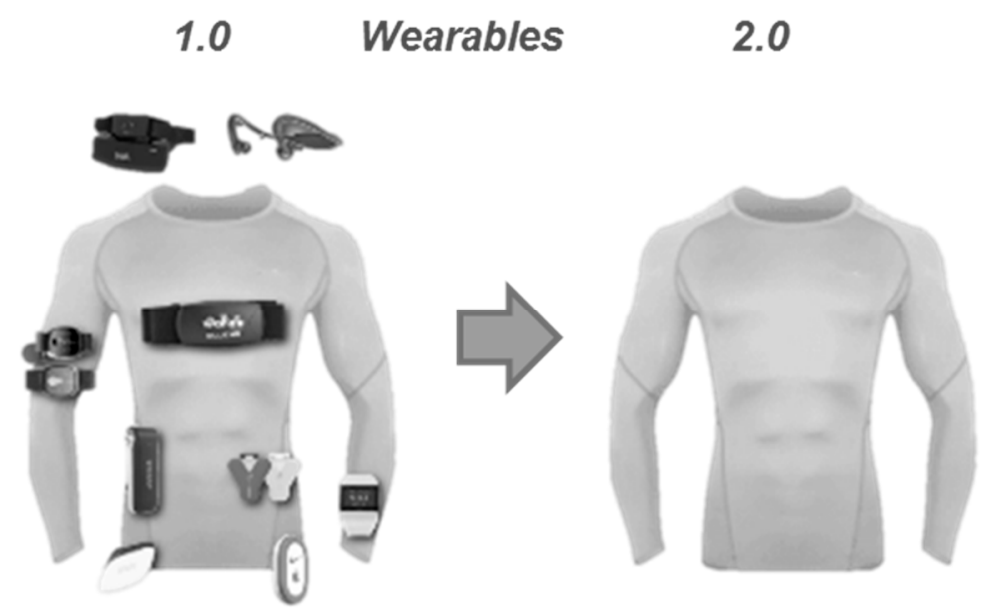

Figure 1. The 1.0 and 2.0 generation of wearable sensors.

In clinical settings WBS would enable monitoring of patients over extensive periods of time, and they can be proposed to offer affordable and interactive healthcare, anyplace, anytime for anyone which is the future of healthcare. Clinical application are probably the higher level ones but matching these requirements a downgrade towards lifestyle or sport monitoring is possible [1-10].

Despite the imagined simplicity in doing wearable systems and specifically sensing garments, a great variety of requirements has to be taken into account. Even in their single obviousness or simplicity in some cases, all of these features or specifications are essentials: sensors and actuators, materials, communication (usually wireless), power supply and management, processing technique (onboard/on external devices, off line/real-time), GUI, algorithms for signal processing, connectors, washability and related stability of sensors, body positions and wearability (tasks, sensors, anthropometry...), elasticity and adherence of the supporting platform (garments or belts, or adhesive patch or other), ect..

This paper tries to discuss the most significant aspects to design a sensing wearable platform, in particular related to sensors for physiological signals monitoring.

\section{Wearable System Design: Requirements}

In design wearable systems, we can divide the specifications in two main macro-areas: technological issues and design issues. To the first class belong the requirements related to technology (sensing principle and corresponding sensor implementation, materials and their properties, data transmission, power supply, processing and related algorithms); the second category comprehends specifically physical and design related factors such as anthropometry and gender issues, GUI, body positions and wearability, elasticity and adherence of the body fixing element or of the garments. 


\subsection{Technological Specifications of Wearable Systems.}

Wearable systems are mainly dedicated to non-invasive biosignals (invasive measurements applied to biomedical devices and form strictly medical purposes) that can be collected onto the body surface (usually the skin or the hair) through specific sensors accordingly designed and built on the physical nature of the signal to be measured [11]. We can distinguish mainly among bioelectric (ECG, EMG, EEG, EOG, ENG, and some others), thermal (temperature and its map on the body surface), mechanical (motion of body segments, pressure at the human surface interface), optical (SpO2 or NIRS measurements) or chemical (sweat composition, inhaled/exhaled air composition) signals (a simplified map is reported in Figure 2).

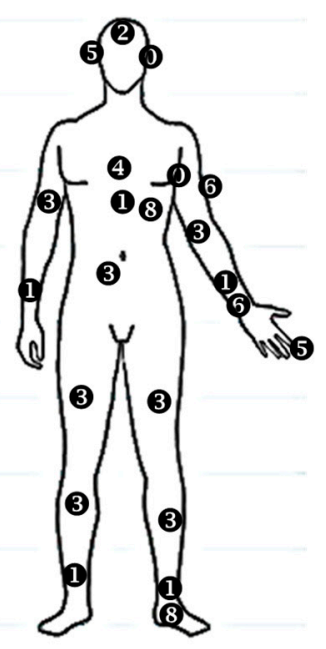

\begin{tabular}{|c|c|c|c|c|}
\hline No. & Signal & Sensor & Parameters & Typology \\
\hline 0 & temperature & piezoresistive & Temperature & Hardware Probe (thermistor) \\
\hline (1) & ECG (ElectroCardioGram) & electrode & Electrical heart activity, Heart Rate & Adhesive, textile, plate \\
\hline$(2)$ & EEG (ElectroEncephaloGram) & electrode & Electrical Brain activity & Plate; textile prototypes \\
\hline 3 & EMG (ElectroMyoGram) & electrode & Electrical muscle activity & Adhesive; textile prototypes \\
\hline (4) & Respiration & $\begin{array}{l}\text { Strain gauge; } \\
\text { Electrode for Impedance measure }\end{array}$ & $\begin{array}{l}\text { Breathing rate, Volumes, respiratory } \\
\text { times }\end{array}$ & $\begin{array}{l}\text { Hardware probe, } \\
\text { Adhesive, textile sensor }\end{array}$ \\
\hline 5 & Blood gas & LED/optical & $\mathrm{SpO} 2, \mathrm{CO} 2$, Heart rate & Hardware Probe, POF for signal transmission \\
\hline 6 & Blood Pressure & Cuff & Systolic/diastolic values, Heart rate & Hardware System \\
\hline 8 & Interface pressure & Piezoresistive; capacitive & Contact pressure & $\begin{array}{l}\text { Piezoresistive ink, capacitive sensor both electric } \\
\text { and textile }\end{array}$ \\
\hline 8 & Resistance & Electrodes for Impedance measure & GSR, Body impedance & Hardware System \\
\hline
\end{tabular}

Note. Movement is measured with miniaturized hardware system applied to each body district.

Figure 2. A simplified map of the biosignals to be measured from the human body through wearables systems.

All of these measures requires the sensor to be closely and firmly in contact with the body and without moving over the skin. For bioelectric signals, sensors are electrodes: in the 2.0 vision these electrodes are constituted by textile conductive parts embedded into clothes is specific positions. The traditional adhesive $\mathrm{Ag} / \mathrm{AgCl}$ electrodes placed directly onto the body in specific positions and with a connecting snap and are non-polarized electrodes as they allow current to pass across the interface between the electrolyte and the electrode [12]. Non-polarized electrodes are better than polarized electrodes in terms of their rejection of motion artifacts and their response to defibrillation currents. Instead textile electrodes (sometimes also called textrodes) belong to the polarized electrodes class; they are a textile structure constituted of electrically conductive yarns which may be used in direct contact with the skin. Textrodes are made of textile yarns with electrical properties: a) Metal yarns, i.e. yarns containing conductive fibers like stainless steel, copper or silver mixed with natural or synthetic fibers; b) Yarns containing electro-conductive fibers like polymeric or carbon coated threads [13]. The main advantage of dry textrodes is the non-irritating and integrating qualities. A disadvantage is the poor skin/electrode contact which is translated into the dedicated design of the embedding garment and/or higher demands on the electronic circuit. Therefore the analogue front-end had to be redesigned and optimised. Additionally perspiration or wetting "storage" capability could help the quality of the skin-electrode contact. This 
effect could be obtained thanks to small sponge under the sensing surface or filling the space underneath the sensor with hydrophilic yarns; this process also produces a convex sensor surface increasing the skin contact pressure so helping the sensor to stay in a fixed position onto the skin itself. The following table 1 summarizes the materials used in textrodes with Pros and Cons.

Table 1. Materials for textile electrodes and related properties.

\begin{tabular}{|c|c|c|}
\hline Material & Merits & Demerits \\
\hline Conductive rubber & $\begin{array}{l}\text { High conductivity, easy to shape, } \\
\text { cheap }\end{array}$ & $\begin{array}{l}\text { Poor flexibility and permeability } \\
\text { to air and liquid }\end{array}$ \\
\hline Silver-coated polymer foam & $\begin{array}{l}\text { High conductivity, easy to shape, } \\
\text { flexible, antibacterial }\end{array}$ & $\begin{array}{c}\text { Poor washability and permeability } \\
\text { to air and liquid, possible } \\
\text { oxidation }\end{array}$ \\
\hline Metal-coated or sputtered fabrics & High conductivity, fabric material & $\begin{array}{l}\text { Poor washability, possible } \\
\text { oxidation }\end{array}$ \\
\hline Woven metal fabric & $\begin{array}{l}\text { Controlled conductivity, fabric } \\
\text { material }\end{array}$ & $\begin{array}{r}\text { Difficult to handle, } \\
\text { low elast }\end{array}$ \\
\hline Woven conductive polymer fabric & Fabric material, elasticity & Low conductivity \\
\hline Carbon yarns & $\begin{array}{l}\text { High mechanical resistance, high } \\
\text { thermal insulation }\end{array}$ & $\begin{array}{l}\text { Average conductivity, skin } \\
\text { irritation, low elasticity }\end{array}$ \\
\hline Stainless steel yarns & $\begin{array}{l}\text { High conductivity, no skin } \\
\text { interaction }\end{array}$ & Low elasticity, high weight \\
\hline
\end{tabular}

The choice of the material strongly determine also requirements like elasticity, washability and chemical agents effects for preserving sensors' efficacy and reliability, and assuring the proper duration of the product for the complete life cycle. This is crucial for sensor reliability or to identify a re-calibration procedure to be carried out at preset times to maintain the required accuracy in measurements.

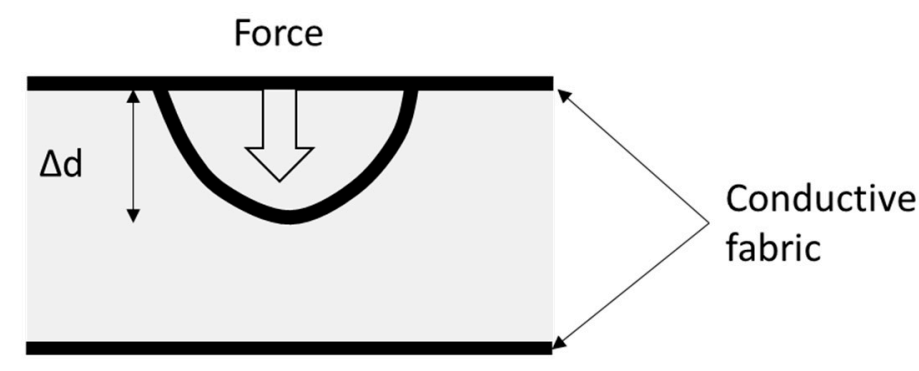

Dielectric substance: capacitance $\hat{\imath}$ Piezoresistive substance: Resistance $\sqrt{ }$

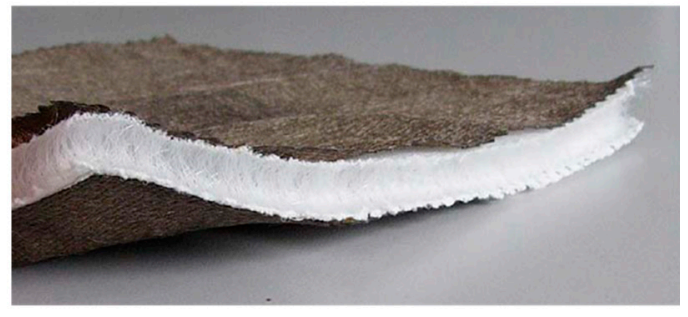

Capacitive textile sensor

Figure 3. A description and an example of capacitance sensor built with conductive fabric.

The choice of the material for sensors also influence the quality and the effects of the skin-sensor contact and interface. Physiological issues are mainly due to skin conductance (and related operations: cleaning, scrubbing the external layer of dead skin cells, the presence of hair-especially in male subjects), skin tolerance and irritation, and the effect of sweat and perspiration. Traditional adhesive electrodes are always used in combination with $\mathrm{Ag} / \mathrm{AgCl}$ gel to establish a good conductive contact with the skin, consequently improving the output signal. However, many patients experience some discomfort since this electrogel may cause skin irritation and softening. These inconveniences impose restrictions 
on the use of this kind of electrode for long-term monitoring. Using textrodes can overcome these limitations because the textile material is in direct contact with the skin hence making electrogel unnecessary and the same effect is obtained after few minutes thanks to the sweat (perspiration). In fact in textrodes usage, the influence of sweat is experienced as a benefit since it is an electrolyte thus improving the conductivity of the electrical signal towards the electrode.

Textile structures containing electroconductive material, are useful as strain gauges when they are carefully engineered and characterized; in this case they are suitable for measuring mechanically induced elongations (from motion of body structures) and with proper calibration they can provide satisfying accuracy in measuring breathing parameters or joints' movements (Figure 4).
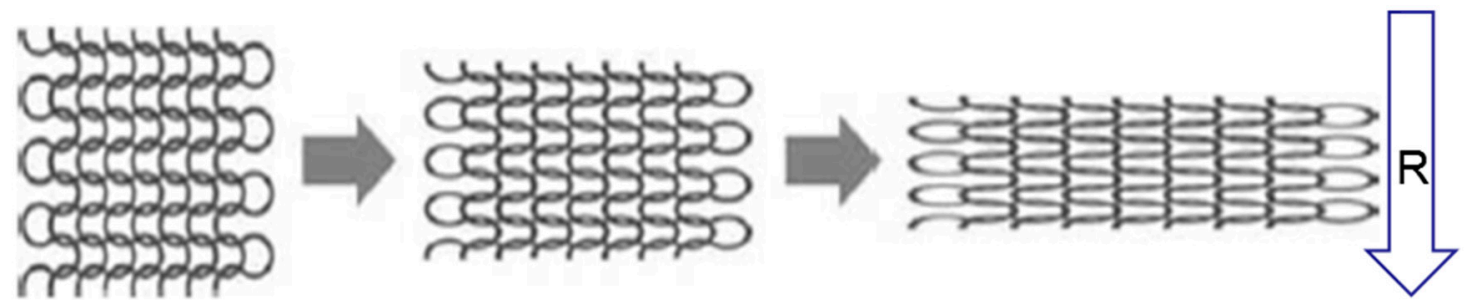

A strain gage exhibits a percent change in resistance that is directly proportional to the strain applied.

Strain $=d L / L \quad R / R=S_{g}{ }^{*}$ Strain

Gage factor $=S_{g}$ is the coefficient to convert strain to $d R / R$

(a)

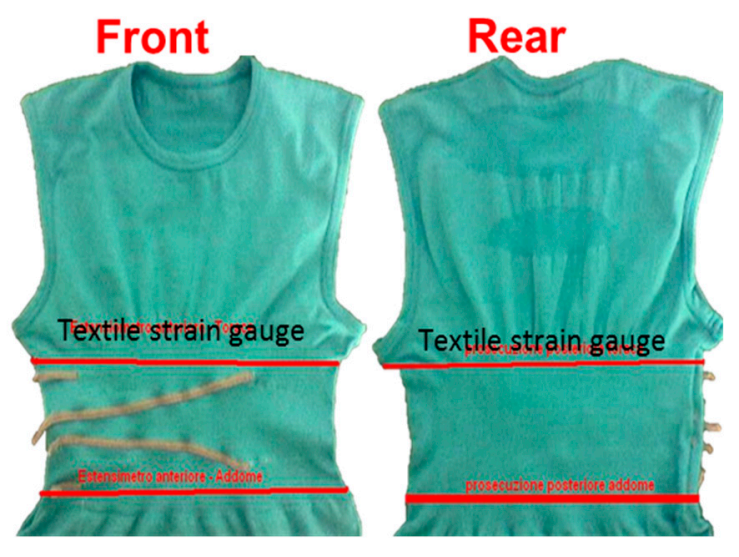

(b)

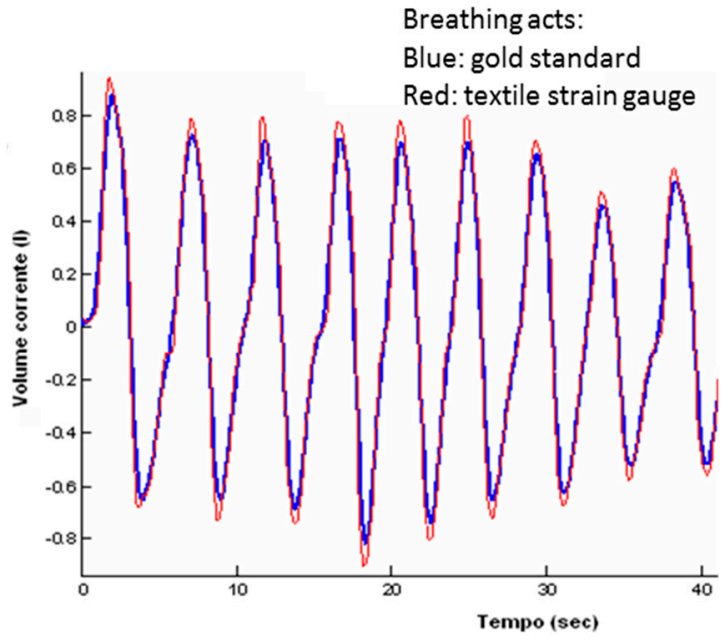

(c)

Figure 4. (a) A model of a textile strain gauge and the related description. (b) A sensorized $\mathrm{t}$-shirt for monitoring respiration through textile strain gauges at thorax and abdominal level. (c) the comparison of the signals from the textile sensor and the pneumotachograph (gold standard technique): $\sim 5 \%$ air volume overestimation at peaks.

\subsection{Design Specifications of Wearable Systems.}

In designing wearable sensor anthropometric and gender considerations are the first ones to be included into design. 
Anthropometry is the discipline dealing with the measurement of physical features of the human body and their differences related to age, gender, lifestyle and ethnicity as inter-subject variability factors, or even time (from circadian to long period) and external environment (e.g. microgravity as extreme condition) as intra-subjective factors [14]. Designing garments for man and woman have obvious different requirements for shape and fit not only for their different dimension but also for the daily task the users have to do. This aspect determine the best sensors' placement: for example if the most used electrode configuration for heart electrical activity monitoring is the 2-sensors setup on the right and on the left side of the chest in correspondence of the 10th rib, if the subject performs important upper limbs movements during the monitoring period, EMG artefacts produced by pectoral muscles could significantly affect the quality and the reliability of the ECG signal. Thus a transversal electrode configuration could be recommendable and specific sensing garment design required (Figure 5). Its specific and modulated elasticity along the different part of the garment is needed to assure a good sensor-skin contact and to minimize their movements over the skin during the recording [15]. This phenomenon known also as skin motion artifact in biomechanical analysis applied to wearable monitoring as well and its avoidance or minimization is crucial to have a good signal for most of the measuring time. Because of the intrinsic weakness of the potential of the heart measurable at the skin $(0.5-2 \mathrm{mV})$, a close contact between electrodes and skin is of major importance. Therefore elasticity of the garment is a highly required property, improving close fitting of the suit around the thorax. Modulated elasticity is obtained by proper choice of elastic yarns and/or by design, and they determine also comfort and wearability of the garments itself. Wearability is crucial for weak users (e.g., elderly, patient under rehabilitation, ...) for autonomous operations and repeatable sensors inter-session repositioning to achieve a reliable monitoring.

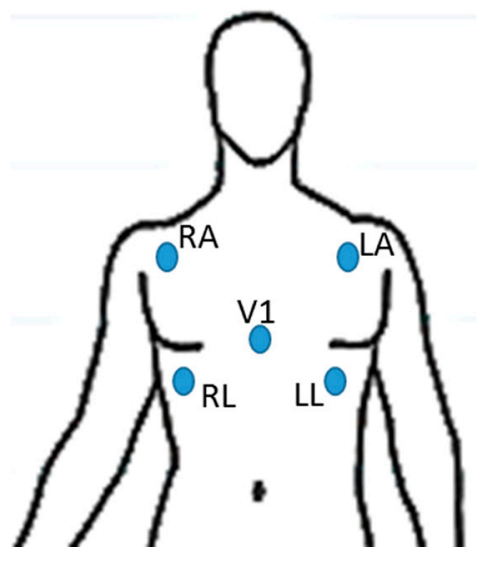

(a)

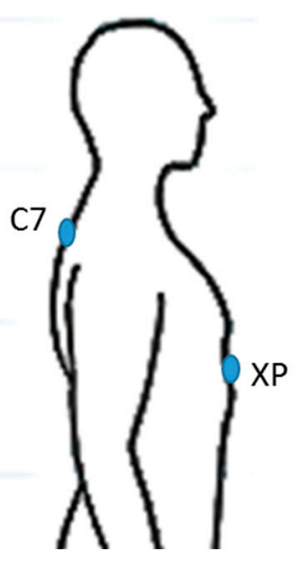

(b)

Figure 5. (a) The standard sensor position for ECG measurements for the reduced 5 leads setup. (b) a transversal ECG sensors setup to avoid pectoralis muscles artefacts $(\mathrm{C} 7=$ in correspondence of the 7 th cervical vertebra, $\mathrm{XP}=$ in correspondence of the xiphoid process).

All these factors are to be integrated with aesthetical requirements are proved to be crucial as well as. For this aspect, user's age, target application, and fashion trend could not be ignored, because they determine the final success of the wearable monitoring approach. Being in close contact with the skin the garment is usually the underwear, so that color, material (cotton vs technical fabrics), fitting, the 
choice of substituting or integrating own underwear (this specifically applies to elderly women that often prefer a sensorized belt to be worn in parallel and with their traditional bra despite to have a new integrated solution) are fundamental aspects to be included in the system design.

Gemperle et al. [16] analyzed the wearability requirements for hardware systems that have been formalized in a 13-points guideline here reported: (1). Placement (where on the body it should go); (2). Form Language (defining the shape); (3). Human Movement (consider the dynamic structure); (4). Proxemics (human perception of space); (5). Sizing (for body size diversity); (6). Attachment (fixing forms to the body); (7). Containment (considering what's inside the form); (8). Weight (as its spread across the human body); (9). Accessibility (physical access to the forms); (10). Sensory Interaction (for passive or active input); (11). Thermal (issues of heat next to the body) (12). Aesthetics (perceptual appropriateness); (13). Long-term Use (effects on the body and mind).

\section{Discussion and Conclusions}

The future of healthcare is based on the development of new technologies and systems for a more accurate, personalized and widespread diagnosis and treatment of pathologies [1]. Among the most relevant innovations, Personal Health Systems (PHS) are a recent concept (late 1990s), that are completely changing the way in which healthcare is provided. PHS have been designed to place the individual citizen/patient in the center of the healthcare delivery process. They allow citizens/patients to have more responsibility in managing their own health and interacting, whenever is necessary, with care providers. In doing so, PHS aim to bring benefits to citizens and health authorities alike: First, by improving the quality of care for the individuals themselves; second, by containing the rising healthcare costs through proper and efficient use of technological capabilities [1,17].

Wearable Biomedical Systems (WBS) are a specific category of PHS: in particular, they can be defined as integrated systems on a wearable platform (in the sense of clothing or devices attachable to the human body) and can offer solutions for continuous monitoring by measuring non-invasive biomedical, biochemical and physical parameters. Continuous monitoring with early detection of anomalies has likely the potential to provide patients with an increased level of confidence, which in turn may improve the quality of life. In addition, ambulatory monitoring will allow patients to engage in practicing normal activities of daily life, rather than staying at home or close to specialized medical services. Thus WBS are an ideal platform for multi-parametric non-intrusive monitoring of health status, thus providing a remote primary and secondary prevention. In this way it is possible to obtain the early diagnosis and management of several diseases (in particular cardiovascular and / or respiratory, but also metabolic pathologies and physical rehabilitation), but also to support elderly and disabled people.

WBS are used to measure a variety of biological signals, including heart rate (or even ECG tracks), electrical cerebral activity (EEG), respiration, blood gases saturation, body accelerations, and many others. For example, in case the necessity is to guarantee a general monitoring of physiological parameters, a good balance between system's complexity and user's compliance might be obtained by a wearable device monitoring 1 or 2 ECG leads, breathing parameters (rate but also in/expiration times and volumes), and global motion (through one or more 3-axial accelerometers or IMUs).

In this paper we have presented some issues related to the design of these systems with specific reference to technological and human factors requirements. One focus are textile electrodes that are the 
main sensor for bioelectric signals (e.g. ECG, EMG, and EEG) and that have been often integrated in wearable systems to replace the standard adhesive silver/silver chloride $(\mathrm{Ag} / \mathrm{AgCl})$ electrodes which are not suitable for long term continuous monitoring for skin irritation problems.

One critical issue that it often left at the end of the design process is the connection between the electronic part and the textile component/sensor. There is the need of a strong and stable connection between the two parts for have a good signal quality during its recording. In some cases, when the number of contacts is low ( 2 or 3 at maximum) a direct connection through stainless steel nickel free snap buttons could be provided. But with an increasing complexity a dedicate connector possibly integrating flexible PCB or flexible/rigid PCB technology has to be thought. This is the best way to optimize the interface between textile and hardware components without compromising the flexibility and easy wearability of the overall system. This requirement allows to respect the methodological approach pursued by wearable systems: non intrusiveness, i.e., a process that does not affect user behavior and is completely transparent to him/her during his/her own daily activities. Only in this case we will obtain actual wearable 2.0 generation system for our health.

\section{Author Contributions}

Giuseppe Andreoni was in charge of the original idea of the paper: he revised the related literature and started proposing, discussing and reviewing the technological and design issues of wearable systems. He contributed in writing the first draft of the paper and he revised the final version.

Carlo E. Standoli contributed in proposing, discussing and reviewing the design issues of wearable systems. He contributed in writing the related parts of the paper and he revised the final version.

Paolo Perego contributed in proposing, discussing and reviewing the technological issues of wearable systems. He contributed in writing the related parts of the paper and he revised the final version.

\section{Conflicts of Interest}

The authors declare no conflict of interest.

\section{References}

1. Lymberis, A., Gatzoulis, L. Wearable Health Systems: from smart technologies to real applications. Proceedings of 28th Annual International Conference of the IEEE Engineering in Medicine and Biology Society, New York City, New York, USA 31-Aug-2006 to 3-Sep-2006, 2006; 6789-6792.

2. De Rossi, D. and Lymberis A. New generation of smart wearable health systems and applications. IEEE Transactions on Information Technology in Biomedicine 2005, 9(3), 293-294.

3. Andreoni, G., Bernabei, M., Perego, P., Barichello, A., Piccini, L. Example of Clinical Applications of Wearable Monitoring System. International Journal of Computer Research 2011, 18(3/4), 323-339.

4. Piccini, L., Ciani, O., Andreoni, G. Chapter 34. New emerging biomedical technologies for homecare and telemedicine applications: the Sensorwear project. In New Developments in Biomedical Engineering, Campolo D., Ed., In-tech: Rijeka, Croatia, 2010; pp. 675-684.

5. Gilsoo Cho, Smart Clothing: Technology and Applications. CRC Press: Boca Raton, FL, USA, 2009. 
6. Muschiato, S., Romero, M., Perego, P., Costa, F., Andreoni, G. Chapter 48. Designing Wearable and Environmental Systems for Elderly Monitoring at Home. In Advances in Social and Organizational Factors, Vink P., Ed., CRC Press: Boca Raton, FL, USA, 2012, pp. 463-469.

7. Bernabei, M., Preatoni, E., Mendez, M., Piccini, L., Porta, M., Andreoni, G. A Novel Automatic Method for Monitoring Tourette Motor Tics Through a Wearable Device, Movement Disorders 2010, 25(12), 1967-1972.

8. Perego, P., Moltani, A., Andreoni, G. Sport monitoring with Smart Wearable System. In Studies in health technology and informatics, pHealth 2012, Proceedings of the 9th International Conference on Wearable Micro and Nano Technologies for Personalized Health, Porto, Portugal, June 26-28, 2012, Blobel B., Pharow P., Sousa F., Eds., IOS Press, 2012, 224-8.

9. Perego, P., Moltani, A., Fusca, M. Andreoni, G. Wearable monitoring systems in pre-term newborns care. In Studies in health technology and informatics, pHealth 2012, Proceedings of the 9th International Conference on Wearable Micro and Nano Technologies for Personalized Health, Porto, Portugal, June 26-28, 2012, Blobel B., Pharow P., Sousa F., Eds., IOS Press, 2012, 203-9.

10. Fanelli, A., Signorini, M.G., Perego, P., Magenes, G., Andreoni G. A smart wearable prototype for fetal monitoring. Proceeding of 4th International Conference on Applied Human Factors and Ergonomics (AHFE). San Francisco, CA, USA, July 21-25, 2012. USA Publishing.

11. Andre, D., Teller, A. Health. Care. Anywhere. Today. Studies in Health Technology and Informatics. 2005, 118, 89-110.

12. Webster, J.G., Ed., Medical Instrumentation Application and Design, 4th ed., John Wiley \& Sons, New York City, New York, USA, 2006.

13. De Rossi, D., Lymberis, A., Eds., Wearable eHealth Systems for Personalised Health Management, IOS Press: Amsterdam, The Netherlands, 2004.

14. Andreoni G. Il Fattore Uomo nel Design, Aracne Editrice, Roma, Italy, 2012.

15. Andreoni G., Fanelli A., Witkowska I., Perego, P., Fusca, M., Mazzola, M., Signorini, M.G. Sensor validation for wearable monitoring system in ambulatory monitoring: application to textile electrodes. Proceedings of the 7th International Conference on Pervasive Computing Technologies for Healthcare, Venice, Italy May 5-8, 2013, ICST (Institute for Computer Sciences, SocialInformatics and Telecommunications Engineering), Ed., pp. 169-175.

16. Gemperle, F., Kasabach, C., Stivoric, J., Bauer, M., Martin, R. Design for Wearability, In ISWC '98, Proceedings of 2nd IEEE International Symposium on Wearable Computers, IEEE Computer Society, Ed. :Washington, DC, USA, 1998.

17. Andreoni, G. Sistemi di sensori indossabili per il monitoraggio: dalla Ricerca al Mercato. In Sistemi indossabili intelligenti per la Salute e la Protezione dell'Uomo; Bonfiglio, A., Cerutti, S., De Rossi, D., Magenes G., Eds.; Patron, Bologna, Italy, 2008; pp. 357-384.

(C) 2015 by the authors; licensee MDPI, Basel, Switzerland. This article is an open access article distributed under the terms and conditions of the Creative Commons Attribution license (http://creativecommons.org/licenses/by/4.0/). 